\title{
OPEN Cross-sectional and longitudinal associations of apolipoprotein A1 and B with glycosylated hemoglobin in Chinese adults
}

\author{
Hongli Dong ${ }^{1,4}$, Wenqing $\mathrm{Ni}^{2,4}$, Yamin $\mathrm{Bai}^{3}$, Xueli Yuan ${ }^{2}$, Yan Zhang ${ }^{2}$, Hongmin Zhang ${ }^{2}$, \\ Yuanying Sun ${ }^{2} \& \operatorname{Jian} X u^{2 \bowtie}$
}

Apolipoproteins exert a key role on glucose metabolism; however, scarce data have examined the relationship between apolipoproteins and glycated haemoglobin (HbA1c) in Chinese adults. This study determined the cross-sectional and longitudinal associations of serum Apolipoprotein A1 (ApoA1), Apolipoprotein B (ApoB) and the ApoB/A1 ratio with $\mathrm{HbA1C}$ in Chinese adults. A total of 1448 subjects ( 584 men and 864 women) aged 54.8 years were included in a baseline survey, and the concentrations of Apo and $\mathrm{HbA1C}$ were measured. A total of 826 participants were followed up approximately once after $3.94 \pm 0.62$ years. In cross-sectional analysis, serum ApoA1 was inversely associated with HbA1c, while $A p o B$ and the ApoB/A1 ratio were positively associated with $\mathrm{HbA1c}$. After further adjusting for the potential covariates, a higher ApoA1 was associated with lower HbA1c (Quartile 4 [Q4] vs. $Q 1=5.673 \%$ vs. $5.796 \%, P$-trend $=0.014)$. In contrast, positive association of $A$ poB concentration and the ApoB/A1 ratio with $\mathrm{HbA1c}$ level were showed (Q4 vs. $\mathrm{Q} 1=5.805 \%$ vs. $5.589 \%$ for $A$ poB; $\mathrm{Q} 4 \mathrm{vs.}$ $\mathrm{Q} 1=5.841 \%$ vs. $5.582 \%$ for $A$ poB $/ A 1$ ratio). The longitudinal results showed no significant associations of ApoA1, ApoB levels and the ApoB/A1 ratio with HbA1c changes (all $P$-trends $>0.05$ ). Path analysis suggested that body mass index did not have mediating effect on Apo-HbA1c association. Our findings revealed that higher $A$ poA1, lower $A$ poB concentrations and the $A$ poB/A1 ratio were associated with lower HbA1c level in Chinese adults.

Glycated haemoglobin (HbAlc), an integrated measure of circulating blood glucose levels during the previous 2 to 3 months, is considered as a gold standard for long period follow-up of blood glycemic control ${ }^{1,2}$. Elevated $\mathrm{HbAlc} \mathrm{level} \mathrm{is} \mathrm{one} \mathrm{of} \mathrm{the} \mathrm{predominant} \mathrm{risk} \mathrm{factors} \mathrm{for} \mathrm{diabetes} \mathrm{and} \mathrm{its} \mathrm{complications}{ }^{3}$. Stratton et al. ${ }^{4}$ found that the reduction in updated mean $\mathrm{HbAlc}$ level was beneficial to the reduction in risk of any diabetes-related end point and deaths. Therefore, it is urgent to explore the novel modifiable factors of $\mathrm{HbAlc}$ for the improvement of diabetes and its complications.

Apolipoproteins, a main protein part of lipoproteins, play an important role in the pathological process of type 2 diabetes mellitus ${ }^{5,6}$. Apolipoprotein A1 (ApoA1) is the major lipoprotein associated with high-density lipoprotein cholesterol (HDL-C) and exert a key part in the transfer of cholesterol from the periphery to the liver in the circulation ${ }^{7}$. Apolipoprotein $\mathrm{B}(\mathrm{ApoB})$ is the major protein part of low-, intermediate-, and very lowdensity lipoproteins ${ }^{8}$. In vitro and animal studies have demonstrated anti- or pro-diabetic effects of ApoA1 and $A \mathrm{AoB}^{9-11}$, observational studies aimed at exploring the association of $\mathrm{ApoA} 1$ and $\mathrm{ApoB}$ with $\mathrm{HbA1c}$ level found the inverse or null association with ApoA $1^{12-14}$ and positive association with $A p o B^{13}$. These studies suggest that ApoAl and ApoB may play important roles in maintaining circulating HbAlc level. Previous study indicated that the levels of ApoA, ApoB, and lipoprotein were lower in Chinese adults than in Caucasians ${ }^{15}$, however, scarce data is available for Chinese populations who differ from their Western counterparts in concentrations of lipoprotein ${ }^{12-14}$. Additionally, the different prevalence of obesity between Western and Chinese populations

\footnotetext{
${ }^{1}$ Scientific Education Section and Department of Child Healthcare, Affiliated Maternity \& Child Health Care Hospital of Nantong University, Nantong, China. ${ }^{2}$ Department of Elderly Health Management, Shenzhen Center for Chronic Disease Control, Shenzhen, China. ${ }^{3}$ Center for Chronic and Non-Communicable Diseases Control and Prevention, Chinese Center for Disease Control and Prevention, Beijing, China. ${ }^{4}$ These authors contributed equally: Hongli Dong and Wenqing Ni. ${ }^{\varpi}$ email: anniexu73@126.com
} 


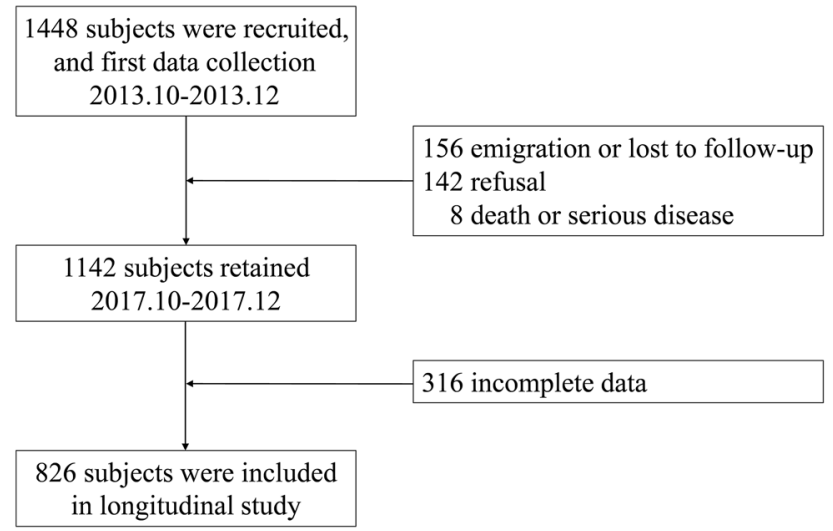

Figure 1. Flow chart of study subjects.

may have impact on the Apo-HbA1c association because body fat can affect the body's glucometabolism ${ }^{16,17}$. Therefore, the Apo-HbAlc association in Chinese populations remains unclear.

Obesity is an important risk factor in maintaining circulating HbAlc level ${ }^{18}$. Previous study demonstrated a significant correlation between Apo levels and obesity ${ }^{19}$. No study, however, examined whether or not the association between Apos and HbAlc is mediated by obesity.

The present study assessed the cross-sectional and longitudinal associations of ApoA1, ApoB levels and the ApoB/A1 ratio with HbAlc concentrations, and examined the mediating effects of body mass index (BMI) in the Apo-HbA1c association in middle-aged and elderly Chinese.

\section{Materials and methods}

Study subjects. A community-based longitudinal study was conducted in Shenzhen, China, during a period from October 2013 to December 2017. 1448 participants aged 54.8 years from October to December 2013 were recruited to complete a baseline survey. ApoA1, ApoB, lipids, and HbA1c concentrations were examined at baseline. The subjects were followed up once in October to December 2017. During the follow-up period, subjects were excluded $(n=306)$ according to the following pre-defined criteria: (1) emigration or lost to followup $(n=156)$; (2) refusal $(n=142)$; (3) death or serious disease $(n=8)$. Finally, 1142 subjects completed their follow-up. We further excluded those with incomplete data $(n=316)$. A total of 826 subjects were included in the follow-up analyses (Fig. 1). HbAlc were examined in this follow-up. The present study was approved by the Shenzhen Center for Chronic Disease Control Human Ethics Committee (No. 20130411) and therefore performed in accordance with the 1964 Declaration of Helsinki and its later amendments. Written informed consent was obtained from all participants.

Questionnaire interview and laboratory assays. Detailed sociodemographic characteristics and health parameters were collected by a structured questionnaire ${ }^{20}$ including age, marriage, sex, education level, alcohol consumption (current drinker or non-drinker), cigarette smoking (current smoker or non-smoker), exercise (sitting, light, moderate, strenuous), lipid-lowering drugs and hypoglycemic medication use. The body height and weight of the participants were measured, and BMI was calculated. Vein blood samples of the participants were collected after 10-14 h of fasting. Serum triglyceride (TG), total cholesterol (TC), HDL-C and low density lipoprotein cholesterol (LDL-C) concentrations were determined using commercial reagents (Olympus System Reagents, Olympus Diagnostica, Ireland) in an autoanalyzer (Olympus AU400 System, Tokyo, Japan). The HbA1c level in the red blood cells was measured by a Bole glycated hemoglobin D-10 kit on a Bole glycated hemoglobin analyzer D-10. Serum ApoA1 and ApoB concentrations were measured based on standardized operation. Briefly, serum sample $(3 \mu \mathrm{L})$ were mixed with the reagent $(240 \mu \mathrm{L})$ composed of polyethylene glycol $(30 \mathrm{~g} / \mathrm{L})$ and phosphate buffer $(0.05 \mathrm{~mol} / \mathrm{L}, \mathrm{pH}=7.0)$. The mixture was vortexed for $2-3 \mathrm{~min}$ and then incubated for $5 \mathrm{~min}$ at $37^{\circ} \mathrm{C}$. The concentrations of ApoAl and $\mathrm{ApoB}$ were detected using the polyethylene glycol-enhanced immunology turbidimetric assay in a 7600-010 automatic analyzer (Hitachi, Japan). The CVs of ApoA1 and ApoB were 3.7 and 4.5\%, respectively. ApoB/A1 ratio was calculated.

Statistical analyses. Categorical- and continuous-variables were described using frequencies (percentages) and means $( \pm S D)$ in both women and men. The mean difference in the continuous variables and the proportion difference among categorical variables were evaluated using Student's t-test and Chi-square test, respectively. According to ApoA1, B levels and the ApoB/A1 ratio, the participants were divided into quartiles, respectively. The lowest quartile defined as the reference. The HbAlc level for the 2 nd-4th quartiles of Apo were described using means and standard error (SE). The mean differences of HbAlc level and the trends in quartiles of Apo were tested using multivariate-analyses of covariance (ANCOVAs). The pair-wise comparisons were examined among quartiles by the Bonferroni test. Univariate analysis was conducted in Model 1 . Sex and age were adjusted in Model 2. BMI, education, marriage, exercise, cigarette smoking, alcohol consumption, hypoglycaemic agent, and lipid-lowering drugs use were further adjusted in Model 3. The mediating effects of BMI on 


\begin{tabular}{|c|c|c|c|}
\hline Variables & Men $(n=584)$ & Women $(n=864)$ & $P^{\text {a }}$ value \\
\hline Age, years & $55.1 \pm 12.00$ & $54.6 \pm 11.44$ & 0.406 \\
\hline BMI, $\mathrm{kg} / \mathrm{m}^{2}$ & $24.34 \pm 3.07$ & $23.84 \pm 3.05$ & 0.002 \\
\hline Education level, n (\%) & & & $<0.001$ \\
\hline Junior high school & $44(7.5)$ & $143(16.6)$ & \\
\hline High school & $280(47.9)$ & $440(50.9)$ & \\
\hline College degree or above & $260(44.5)$ & $281(32.5)$ & \\
\hline Marriage, n (\%) & & & 0.005 \\
\hline Married & $562(96.2)$ & $795(92.0)$ & \\
\hline Unmarried & $4(0.7)$ & $16(1.9)$ & \\
\hline Divorce/widowed & $18(3.1)$ & $53(6.1)$ & \\
\hline Exercise, n (\%) & & & 0.008 \\
\hline Sitting & $423(72.4)$ & $624(72.2)$ & \\
\hline Light & $103(17.6)$ & $152(17.6)$ & \\
\hline Moderate & $34(5.8)$ & $74(8.6)$ & \\
\hline Strenuous & $24(4.2)$ & $14(1.6)$ & \\
\hline Smoker, n (\%) & $201(34.4)$ & $22(2.5)$ & $<0.001$ \\
\hline Alcohol drinker, n (\%) & $131(22.4)$ & $49(5.7)$ & $<0.001$ \\
\hline Lipid-lowering drugs user, n (\%) & $42(7.2)$ & $60(6.9)$ & 0.857 \\
\hline Hypoglycaemic agent user, n (\%) & $19(3.3)$ & $26(3.0)$ & 0.793 \\
\hline $\mathrm{TC}(\mathrm{mmol} / \mathrm{L})$ & $4.97 \pm 0.77$ & $5.04 \pm 0.68$ & 0.042 \\
\hline $\mathrm{TG}(\mathrm{mmol} / \mathrm{L})$ & $1.64 \pm 1.24$ & $1.42 \pm 0.92$ & $<0.001$ \\
\hline $\mathrm{HDL}-\mathrm{C}(\mathrm{mmol} / \mathrm{L})$ & $1.31 \pm 0.52$ & $1.41 \pm 0.37$ & $<0.001$ \\
\hline $\mathrm{LDL}-\mathrm{C}(\mathrm{mmol} / \mathrm{L})$ & $2.90 \pm 0.71$ & $2.87 \pm 0.66$ & 0.343 \\
\hline ApoA1, g/L & $1.43 \pm 0.29$ & $1.56 \pm 0.26$ & $<0.001$ \\
\hline ApoB, g/L & $1.02 \pm 0.24$ & $0.99 \pm 0.23$ & 0.032 \\
\hline ApoB/A1 ratio & $0.77 \pm 0.47$ & $0.65 \pm 0.19$ & $<0.001$ \\
\hline HbAlc, \% & $5.66 \pm 0.66$ & $5.71 \pm 0.65$ & 0.198 \\
\hline
\end{tabular}

Table 1. The baseline characteristics of the study participants. Apo Apolipoprotein, $B M I$ body mass index, $H b A 1 c$ Glycated haemoglobin, $H D L-C$ high density lipoprotein cholesterol, $L D L-C$ low density lipoprotein cholesterol, $T C$ total cholesterol, $T G$ triglycerides. ${ }^{a} P$ values were calculated by Student's $t$ test for the continuous variables and Chi-square test for categorical variables. Significant values are in bold.

Apo-HbA1c association were evaluated using path analyse ${ }^{21}$. Standardized regression coefficients were assessed in each path. SPSS Statistics 21.0 (IBM SPSS Statistics, USA) and SPSS AMOS21.0 (IBM Corporation, USA) were performed to analyze the data. Two-tailed $P<0.05$ was considered statistically significant.

\section{Results}

Characteristics of participants. As shown in Table 1, the mean age of men and women were 55.1 and 54.6 years, respectively. Lower BMI $\left(23.84 \mathrm{~kg} / \mathrm{m}^{2}\right.$ vs. $\left.24.34 \mathrm{~kg} / \mathrm{m}^{2}\right)$, TG $(1.42 \mathrm{mmol} / \mathrm{L}$ vs. $1.64 \mathrm{mmol} / \mathrm{L})$, ApoB $(0.99 \mathrm{~g} / \mathrm{L}$ vs. $1.02 \mathrm{~g} / \mathrm{L})$, and the ApoB/A1 ratio (0.65 vs. 0.77$)$ and higher TC $(5.04 \mathrm{mmol} / \mathrm{L}$ vs. $4.97 \mathrm{mmol} / \mathrm{L})$, HDL-C (1.41 mmol/L vs. $1.31 \mathrm{mmol} / \mathrm{L})$ and ApoA1 (1.56 g/L vs. $1.43 \mathrm{~g} / \mathrm{L})$ were observed in women than in men (all $P<0.05)$.

Partial correlation analysis. Table 2 showed the inverse relationship between ApoA 1 and HbAlc in both models 1 and 2 in partial correlation analysis. In contrast, positive association of ApoB concentration and the ApoB/A1 ratio with HbAlc level were showed (Table 2).

Associations of $\mathrm{ApoA1}, \mathrm{ApoB}$, and the $\mathrm{ApoB} / \mathrm{A1}$ ratio with $\mathrm{HbA1c}$ level. In cross-sectional analysis, serum ApoA1 showed an inverse association with $\mathrm{HbA1c}$, while ApoB and the ApoB/A1 ratio exhibited positive association with HbA1c (Table 3). In Model 1 with univariate analysis, ApoA1 was inversely related to HbAlc $(P$-trend $=0.046)$, whereas positive associations were observed for ApoB level and the ApoB/A1 ratio $(P$-trend $<0.001)$. With adjustment for sex and age in Model 2, ApoA1 concentration was inversely associated with HbA1c level (quartile 4 [Q4] vs. Q1 $=5.671 \%$ vs. $5.801 \%, P$-trend $=0.010$ ). In contrast, ApoB concentration and the ApoB/A1 ratio were positively associated with $\mathrm{HbA1c}$ level (Q4 vs. Q1 $=5.808 \%$ vs. 5.588\% for ApoB; $\mathrm{Q} 4$ vs. Q1 $=5.839 \%$ vs. $5.584 \%$ for ApoB/A1 ratio). After further adjusting for the other potential covariates in Model 3, a higher ApoA1 concentration was associated with lower HbA1c level (Q4 vs. Q1 =5.673\% vs. $5.796 \%, P$-trend $=0.014)$. In contrast, the positive associations of $A p o B$ concentration and the ApoB/A1 ratio with $\mathrm{HbA1c}$ level were showed (Q4 vs. Q1 $=5.805 \%$ vs. $5.589 \%$ for ApoB; Q4 vs. Q1 =5.841\% vs. 5.582\% for 


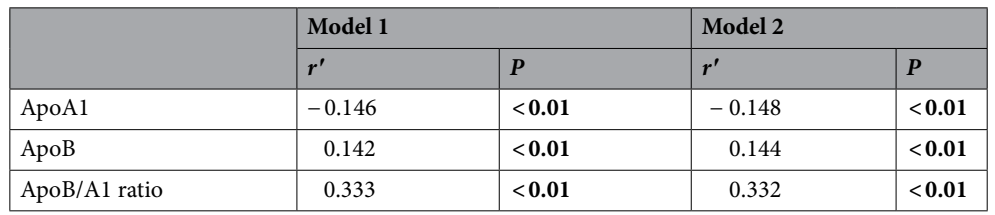

Table 2. Relationships between serum apolipoprotein levels and $\mathrm{HbAlc}$. Abbreviations were shown in Table 1. a Partial correlation analysis, Model 1 adjusted for age and gender. Model 2 adjusted for age, gender, BMI, education, marriage, exercise, cigarette smoking, alcohol consumption, hypoglycaemic agent, and lipidlowering drugs use. Significant values are in bold.

\begin{tabular}{|c|c|c|c|c|c|c|c|}
\hline \multirow{2}{*}{ Variables } & & \multicolumn{4}{|c|}{ Quartiles by apolipoproteins } & \multirow[b]{2}{*}{$P$-diff } & \multirow[b]{2}{*}{$P$-trend } \\
\hline & & Q1 & Q2 & Q3 & Q4 & & \\
\hline \multicolumn{8}{|l|}{ ApoA1 } \\
\hline $\mathrm{n}$ & & 362 & 361 & 357 & 368 & & \\
\hline \multirow{3}{*}{ HbAlc } & Model 1 & $5.783 \pm 0.034$ & $5.640 \pm 0.034^{\mathrm{a}}$ & $5.646 \pm 0.034^{\mathrm{a}}$ & $5.679 \pm 0.034$ & 0.011 & 0.046 \\
\hline & Model 2 & $5.801 \pm 0.034$ & $5.636 \pm 0.034^{\mathrm{a}}$ & $5.640 \pm 0.034^{\mathrm{a}}$ & $5.671 \pm 0.034^{\mathrm{a}}$ & $<0.001$ & 0.010 \\
\hline & Model 3 & $5.796 \pm 0.033$ & $5.638 \pm 0.033^{\mathrm{a}}$ & $5.640 \pm 0.033^{\mathrm{a}}$ & $5.673 \pm 0.033$ & 0.002 & 0.014 \\
\hline \multicolumn{8}{|l|}{ ApoB } \\
\hline $\mathrm{n}$ & & 365 & 368 & 357 & 358 & & \\
\hline \multirow{3}{*}{ HbAlc } & Model 1 & $5.576 \pm 0.034$ & $5.626 \pm 0.034$ & $5.732 \pm 0.034^{\mathrm{a}}$ & $5.819 \pm 0.034^{\mathrm{bc}}$ & $<0.001$ & $<0.001$ \\
\hline & Model 2 & $5.588 \pm 0.033$ & $5.631 \pm 0.033$ & $5.725 \pm 0.034^{\mathrm{a}}$ & $5.808 \pm 0.034^{b c}$ & $<0.001$ & $<0.001$ \\
\hline & Model 3 & $5.589 \pm 0.033$ & $5.631 \pm 0.032$ & $5.728 \pm 0.033^{\mathrm{a}}$ & $5.805 \pm 0.033^{b c}$ & $<0.001$ & $<0.001$ \\
\hline \multicolumn{8}{|c|}{ ApoB/A1 ratio } \\
\hline $\mathrm{n}$ & & 362 & 362 & 362 & 362 & & \\
\hline \multirow{3}{*}{ HbAlc } & Model 1 & $5.578 \pm 0.034$ & $5.614 \pm 0.034$ & $5.719 \pm 0.034^{\mathrm{a}}$ & $5.838 \pm 0.034^{b c}$ & $<0.001$ & $<0.001$ \\
\hline & Model 2 & $5.584 \pm 0.033$ & $5.621 \pm 0.033$ & $5.704 \pm 0.033$ & $5.839 \pm 0.033^{\text {bcd }}$ & $<0.001$ & $<0.001$ \\
\hline & Model 3 & $5.582 \pm 0.033$ & $5.630 \pm 0.033$ & $5.696 \pm 0.033$ & $5.841 \pm 0.033^{\text {bcd }}$ & $<0.001$ & $<0.001$ \\
\hline
\end{tabular}

Table 3. Mean HbA1c level according to quartiles of apolipoprotein in all participants (mean $\pm \mathrm{SE}$ ). Apo apolipoprotein, $H b A 1 c$ glycosylated hemoglobin, Q quartile. P-Diff: Multiple comparison among quartiles. Model 1 was univariate analysis. Model 2 was adjusted for sex, age. Model 3 further adjusted for BMI, education, marriage, exercise, cigarette smoking, alcohol consumption, hypoglycaemic agent, and lipidlowering drugs use. ${ }^{a} P<0.05$ compared with Q1. ${ }^{\mathrm{b}} P<0.001$ compared with Q1. ${ }^{c} P<0.001$ compared with Q2. ${ }^{\mathrm{d}} P<0.05$ compared with Q3. Significant values are in bold.

ApoB/A1 ratio). Additionally, we found null associations of ApoA1, ApoB levels and the ApoB/A1 ratio with HbAlc changes across the three models (all $P$-trends $>0.05$ ) (Table 4 ).

Associations of serum lipids levels with HbA1c level. As shown in Supplementary Table S1, a higher serum HDL-C was associated with lower HbA1c. In contrast, higher serum TG and TC concentration were associated with higher HbA1c level across the three models. Null association between serum LDL-C with HbA1c level was detected. At follow-up, no significant associations were observed between ApoA1, ApoB levels and the ApoB/A1 ratio with $\mathrm{HbA1c}$ changes (all $P$-trends $>0.05$ ) (Supplementary Table S2).

Path analysis. Path analysis was used to assess whether BMI mediated the associations of ApoA1, ApoB levels and the ApoB/A1 ratio with HbA1c. As shown in Fig. 2, path analyses indicated that BMI did not have direct effect on HbAlc. Mediating effects of BMI on the Apo-HbAlc association were not found in subjects (Fig. 2).

\section{Discussion}

To the best of our knowledge, the present study firstly identified the potential influence of ApoA1, ApoB concentrations and the $\mathrm{ApoB} / \mathrm{A} 1$ ratio on $\mathrm{HbA1c}$ level in middle-aged and elderly Chinese populations. Our results showed that a higher serum ApoAl concentration was associated with lower $\mathrm{HbAlc}$ level, while higher ApoB concentration and ApoB/A1 ratio were associated with higher HbA1c level. There was no evidence of the mediating effects of BMI on the Apo-HbAlc associations.

ApoA1 and HbA1c level. ApoA1, a major protein component of HDL, has been shown to have a distinct effect on glucose metabolism ${ }^{22}$. The protective effect of HDL was partly attributed to ApoA $1^{23}$. Our results demonstrated that both ApoA1 and an intermediate HDL-C levels were inversely associated with HbA1c. An in vivo and in vitro study showed that ApoA1 could improve glucose metabolism by attenuating ability of catalyzing 


\begin{tabular}{|c|c|c|c|c|c|c|c|}
\hline \multirow[b]{2}{*}{ Variables } & & \multicolumn{4}{|c|}{ Quartiles by apolipoproteins } & \multirow[b]{2}{*}{$P$-diff } & \multirow[b]{2}{*}{$P$-trend } \\
\hline & & Q1 & Q2 & Q3 & Q4 & & \\
\hline \multicolumn{8}{|l|}{ ApoA1 } \\
\hline $\mathrm{n}$ & & 208 & 203 & 211 & 204 & & \\
\hline \multirow{3}{*}{ HbAlc } & Model 1 & $0.028 \pm 0.047$ & $-0.126 \pm 0.048$ & $-0.049 \pm 0.047$ & $0.026 \pm 0.048$ & 0.069 & 0.732 \\
\hline & Model 2 & $0.029 \pm 0.047$ & $-0.126 \pm 0.048$ & $-0.050 \pm 0.047$ & $0.026 \pm 0.048$ & 0.068 & 0.760 \\
\hline & Model 3 & $0.036 \pm 0.047$ & $-0.118 \pm 0.048$ & $-0.054 \pm 0.047$ & $0.015 \pm 0.048$ & 0.091 & 0.998 \\
\hline \multicolumn{8}{|l|}{ ApoB } \\
\hline $\mathrm{n}$ & & 209 & 205 & 207 & 205 & & \\
\hline \multirow{3}{*}{ HbAlc } & Model 1 & $-0.024 \pm 0.047$ & $-0.014 \pm 0.048$ & $-0.038 \pm 0.048$ & $-0.045 \pm 0.048$ & 0.968 & 0.683 \\
\hline & \begin{tabular}{|l|} 
Model 2 \\
\end{tabular} & $-0.022 \pm 0.048$ & $-0.014 \pm 0.048$ & $-0.038 \pm 0.048$ & $-0.046 \pm 0.048$ & 0.964 & 0.656 \\
\hline & Model 3 & $-0.027 \pm 0.047$ & $-0.015 \pm 0.048$ & $-0.031 \pm 0.047$ & $-0.048 \pm 0.048$ & 0.969 & 0.708 \\
\hline \multicolumn{8}{|c|}{ ApoB/A1 ratio } \\
\hline $\mathrm{n}$ & & 206 & 207 & 207 & 206 & & \\
\hline \multirow{3}{*}{$\mathrm{HbAlc}$} & Model 1 & $-0.042 \pm 0.048$ & $-0.057 \pm 0.048$ & $0.009 \pm 0.048$ & $-0.031 \pm 0.048$ & 0.786 & 0.640 \\
\hline & Model 2 & $-0.041 \pm 0.048$ & $-0.057 \pm 0.048$ & $0.008 \pm 0.048$ & $-0.031 \pm 0.048$ & 0.796 & 0.653 \\
\hline & Model 3 & $-0.050 \pm 0.048$ & $-0.065 \pm 0.047$ & $0.019 \pm 0.048$ & $-0.024 \pm 0.048$ & 0.621 & 0.455 \\
\hline
\end{tabular}

Table 4. Changes in HbAlc level over $\sim 4$ year by quartiles of apolipoprotein in all participants (mean \pm SE). Apo apolipoprotein, $H b A 1 c$ glycosylated hemoglobin, Q quartile. P-Diff: Multiple comparison among quartiles. Model 1 was univariate analysis. Model 2 was adjusted for sex, age. Model 3 further adjusted for BMI, education, marriage, exercise, cigarette smoking, alcohol consumption, hypoglycaemic agent, and lipidlowering drugs use.
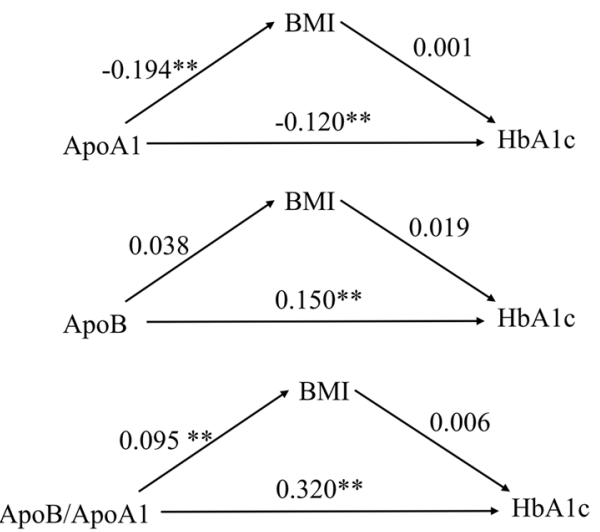

Figure 2. Path analyses of associations of ApoA1, ApoB levels, the ApoB/A1 ratio, mediator (BMI) with $\mathrm{HbA1c}$ in subjects. Apo Apolipoprotein, $B M I$ body mass index, $H b A 1 c$ glycated haemoglobin; ${ }^{* *} P<0.05$.

cholesterol efflux alter protein structure and decreasing lipid binding capability ${ }^{24}$. This effect was confirmed in a cross-sectional study involving 137 women and 111 men $^{12}$, Sosenko et al. reported that ApoA1 was inversely related to HbA1c level in both men and women $\left(r_{\text {women }}=-0.25\right.$ and $\left.r_{\text {men }}=-0.30\right)$. Nevertheless, in a cross-sectional study with 17,661 participants within the EPIC-Norfolk cohort study, Julian et al. found null associations between serum ApoA1 and HbA1c level ${ }^{25}$. Likewise, Boris et al. and Schauer et al. also reported that ApoA1 was not related to $\mathrm{HbA1c}$ level ${ }^{13,14}$. Some factors might provide explanation for these discrepancies. The favorable association was more readily to be observed in persons with higher HbAlc level (5.69\% [this study] and $6.37 \%{ }^{12}$ vs. $5.3 \%{ }^{25}$ ), in large sample size (1448 subjects [this study] vs. $44^{13}$ and 238 subjects ${ }^{26}$ ). Lipid values formed part of the inclusion criteria, intensity of glycemic therapy and residual insulin secretion might also limit variability in Apo in an early type 2 diabetic population ${ }^{14}$. More large prospective studies are needed to confirm our results. Other reasons, such as the differences in the adjusted covariates and analysis method (e.g., multiple regression analyses $^{12}$, student's t-test ${ }^{13}$, partial correlations ${ }^{14}$, linear regression analyses ${ }^{25,26}$ and analyses of covariance [this study]), might also explain the differences in these studies.

ApoB, ApoB/A1 ratio and HbA1c level. ApoB is the main lipoprotein associated with LDL and lipoprotein(a) particles ${ }^{27}$. Our study observed that ApoB, but not LDL-C was positively associated with $\mathrm{HbAlc}$. Ley et al. pointed out that plasma ApoB was superior to LDL-C in predicting the diseases in Canadian population $^{28}$. There is growing interest in the health benefits of ApoB. Although previous studies have shown 
the unfavorable roles of $\mathrm{ApoB}$ and the $\mathrm{ApoB} / \mathrm{A} 1$ ratio in the cerebrovascular diseases and diabetes ${ }^{5,29}$, scarce data assessed the associations of $A p o B$ level and $A p o B / A 1$ ratio with HbA1c. Based on data from 44 diabetics who participated in the Erfurt Study, higher ApoB level was observed in HbA1c $\geq 8 \%$ group than in $\mathrm{HbAlc}<8 \%$ group $^{13}$. Consistent with this finding, we also observed the positive associations of ApoB and the ApoB/A1 ratio with HbAlc in Chinese adults. Nevertheless, Mustapha et al. ${ }^{26}$ found no significant differences in ApoB/A1 ratio among different $\mathrm{HbA1c}$ groups in both men and women with type 2 diabetes. Hypoglycemic drugs using might attenuate the association between $\mathrm{ApoB} / \mathrm{A} 1$ ratio and $\mathrm{HbA} 1 \mathrm{c}$ in 238 type 2 diabetic patients ${ }^{26}$. Additionally, previous study pointed out that $\mathrm{ApoB} / \mathrm{A} 1$ ratio exerted significant information for predicting insulin resistance $\mathrm{e}^{30}$. Our study also indicated that $\mathrm{ApoB} / \mathrm{A} 1$ ratio might act as a more precise marker for HbA1c measures (ApoA1: $(\mathrm{Q} 4-\mathrm{Q} 1) / \mathrm{Q} 1=-0.021 ; \mathrm{ApoB}:(\mathrm{Q} 4-\mathrm{Q} 1) / \mathrm{Q} 1=0.039 ; \mathrm{ApoB} / \mathrm{A} 1$ ratio: $(\mathrm{Q} 4-\mathrm{Q} 1) / \mathrm{Q} 1=0.046)$.

Potential mechanisms. The associations of ApoAl and ApoB with HbAlc might be explained by various mechanisms. First, ApoA1 may improve glucose tolerance by adenosine monophosphate-activated protein kinasecomplex and increase glucose uptake into skeletal muscle and heart ${ }^{31}$. In addition, ApoA1 can increase insulin sensitivity in skeletal muscle and adipose tissue ${ }^{10}$ and reduce lipid binding capability ${ }^{24}$. Second, dysregulation of ApoB metabolism can induce insulin resistance ${ }^{32}$ and inhibit lipolysis from liver to peripheral fat via acting as a lipid metabolic pathway ${ }^{33}$. However, our path analysis showed no mediating effects of BMI on Apo$\mathrm{HbA1c}$ association. Other inflammation and oxidative stress markers might be mediated in the associations of ApoA1 and ApoB with HbA1c. Finally, ApoA1 can inhibit inflammation via decreasing plasma malondialdehyde level and clearing pro-inflammatory lipids ${ }^{10}$. ApoB can aggravate inflammation by releasing inflammatory cytokines (e.g., IL- $1 \beta$ and tumor necrosis factor- $\alpha)^{34}$ and binding to enolase- 1 . Nevertheless, the detailed mechanism underlying the associations of ApoA1 and ApoB with $\mathrm{HbA} 1 \mathrm{c}$ remains unclear and warrants further investigation.

Strengths and limitations. This study has several strengths. Primarily, to the best of our knowledge, this is the first report that evaluated the associations of serum ApoA1, ApoB and the ApoB/A1 ratio with HbA1c in both cross-sectional and prospective analyses, and examined the mediating effects of BMI on Apo-HbA1c association in Chinese adults. Next, the relatively large sample size provided us with sufficient power to evaluate potential associations among variables. Third, the availability of individual information (e.g., basic characteristics, lifestyles and medication records) allowed us to avoid potential confounding effects. Finally, the probability of falsely significant founds was reduced by assessing different Apo and lipid indices. Notwithstanding, our study also had a few limitations. First, the lack of association between Apo and HbAlc changes in our longitudinal analyses. This might partly be explained by the observed small longitudinal changes in HbAlc [mean $( \pm$ SEM) HbAlc changes: $-0.030 \pm 0.024$ ], which accounted for $<1 \%$ of the mean HbAlc. The small changes in HbAlc would also substantially attenuate the Apo-HbAlc association, particularly in the longitudinal studies. The effect size might be substantially underestimated in the present study, and that is the reason (at least in part) why the Apo-HbAlc associations were much more significant in the cross-sectional data than in their longitudinal counterparts. Thus, the effects of Apo need to be clarified in further prospective studies. Second, the effects of dietary nutrients were not excluded, which might attenuate the underlying associations due to dietary nutrients were associated with Apo concentrations $s^{35}$. Third, as with any observational study, residual confounding could not be ruled out due to the potential confounders. Finally, we could not rule out the possibility of selection bias because our study subjects were not a random sample of Chinese community population. However, we did not found any significant interactions between Apo and age, sex, exercise, smoking, or alcohol drinking on HbA1c. The generalizability of the findings was unlikely to be influenced by these factors.

\section{Conclusion}

Our cross-sectional findings suggested that higher ApoA1, lower ApoB concentrations and the ApoB/A1 ratio were associated with lower $\mathrm{HbAlc}$ level in Chinese adults. More large prospective studies with the longer followup period are needed to clarify the effect of Apo on HbA1c changes.

\section{Data availability}

All data generated or analysed during this study are included in this published article (and its Supplementary Information files).

Received: 13 August 2021; Accepted: 31 January 2022

Published online: 17 February 2022

\section{References}

1. American Diabetes Association. Diagnosis and classification of diabetes mellitus. Diabetes Care 33(Suppl), S62-69 (2010).

2. Longo, D. L. et al. Harrison's Principles of Internal Medicine 18th edn. (The McGraw Hill, 2013).

3. Luo, J., Qu, Y., Zhang, Q., Chang, A. M. \& Jacober, S. J. Relationship of glucose variability with glycated hemoglobin and daily mean glucose: A post hoc analysis of data from 5 phase 3 studies. J. Diabetes. Sci. Technol. 12, 325-332 (2018).

4. Stratton, I. M. et al. Association of glycaemia with macrovascular and microvascular complications of type 2 diabetes (UKPDS 35): Prospective observational study. BMJ (Clin. Res. Ed). 321, 405-412 (2000).

5. Gao, L., Zhang, Y. J., Wang, X. M. \& Dong, H. L. Association of apolipoproteins A1 and B with type 2 diabetes and fasting blood glucose: A cross-sectional study. BMC. Endocr. Disord. 21, 59 (2021).

6. Zhang, P., Gao, J., Pu, C. \& Zhang, Y. Apolipoprotein status in type 2 diabetes mellitus and its complications (review). Mol. Med. Rep. 16, 9279-9286 (2017).

7. van der Vorst, E. P. C. High-density lipoproteins and apolipoprotein A1. Subcell. Biochem. 94, 399-420 (2020). 
8. Andersen, L. H., Miserez, A. R., Ahma, Z. \& Andersen, R. L. Familial defective apolipoprotein B-100: A review. J. Clin. Lipidol. 10, 1297-1302 (2016).

9. Tang, S. et al. Apolipoprotein A-I enhances insulin-dependent and insulin-independent glucose uptake by skeletal muscle. Sci. Rep. 9,1350 (2019).

10. Wu, B. J. et al. Apolipoprotein A-I protects against pregnancy-induced insulin resistance in rats. Arterioscler. Thromb. Vasc. Biol. 39, 1160-1171 (2019).

11. Zhang, X. et al. Genetic polymorphism of APOB is associated with diabetes mellitus in sickle cell disease. Hum. Genet. 134, 895-904 (2015).

12. Sosenko, J. M. et al. High-density lipoprotein and glycosylated hemoglobin in nondiabetic individuals. Arch. Intern. Med. 146, 1521-1524 (1986).

13. Schauer, U. J. et al. Serum lipids and apolipoproteins in relation to glycaemic control and diabetic nephropathy in long-term survivors of diabetes: results of the Erfurt Study. Exp. Clin. Endocrinol. 92, 280-286 (1988).

14. Waldman, B. et al. HDL-C and HDL-C/ApoA-I predict long-term progression of glycemia in established type 2 diabetes. Diabetes Care 37, 2351-2358 (2014).

15. Pare, G. et al. Lipoprotein(a) levels and the risk of myocardial infarction among 7 ethnic groups. Circulation 139, 1472-1482 (2019).

16. Ng, M. et al. Global, regional, and national prevalence of overweight and obesity in children and adults during 1980-2013: A systematic analysis for the Global Burden of Disease Study 2013. Lancet (London, England). 384, 766-781 (2014).

17. Yoon, K. H. et al. Epidemic obesity and type 2 diabetes in Asia. Lancet 368, 1681-1688 (2006).

18. Gummesson, A., Nyman, E., Knutsson, M. \& Karpefors, M. Effect of weight reduction on glycated haemoglobin in weight loss trials in patients with type 2 diabetes. Diabetes. Obes. Metab. 19, 1295-1305 (2017).

19. Su, X. \& Peng, D. The exchangeable apolipoproteins in lipid metabolism and obesity. Clin. Chim. Acta. 503, 128-135 (2020).

20. Ni, W. et al. Clustering of cardiovascular disease biological risk factors among older adults in Shenzhen City, China: A crosssectional study. BMJ Open 9, e024336 (2019).

21. Baron, R. M. \& Kenny, D. A. The moderator-mediator variable distinction in social psychological research: Conceptual, strategic, and statistical considerations. J. Pers. Soc. Psychol. 51, 1173-1182 (1986).

22. Fritzen, A. M. et al. ApoA-1 improves glucose tolerance by increasing glucose uptake into heart and skeletal muscle independently of AMPKa(2). Mol. Metab. 35, 100949 (2020).

23. Fruchart, J. C. Lipoprotein heterogeneity and its effect on apolipoprotein assays. Scand. J. Clin. Lab. Investig. Suppl. 198, 51-57 (1990).

24. Domingo-Espin, J., Nilsson, O., Bernfur, K., Del Giudice, R. \& Lagerstedt, J. O. Site-specific glycations of apolipoprotein A-I lead to differentiated functional effects on lipid-binding and on glucose metabolism. Biochim. Biophys. Acta. Mol. Basis. Dis. 9, 23 (2018).

25. Van Capelleveen, J. C. et al. Association of high-density lipoprotein-cholesterol versus apolipoprotein A-I with risk of coronary heart disease: The European prospective investigation into cancer-norfolk prospective population study, the atherosclerosis risk in communities study, and the women's health study. J. Am. Heart. Assoc. 6, e006636 (2017).

26. Diaf, M., Khaled, B. M. \& Sellam, F. Impact of corpulence parameters and haemoglobin A1c on metabolic control in type 2 diabetic patients: Comparison of apolipoprotein B/A-I ratio with fasting and postprandial conventional lipid ratios. Libyan. J. Med. 10, 27400 (2015).

27. Barter, P. J. et al. Apo B versus cholesterol in estimating cardiovascular risk and in guiding therapy: Report of the thirty-person/ ten-country panel. J. Intern. Med. 259, 247-258 (2006).

28. Ley, S. H. et al. Association of apolipoprotein B with incident type 2 diabetes in an aboriginal Canadian population. Clin. Chem. 56, 666-670 (2010).

29. Dong, H. L. et al. Apolipoprotein A1, B levels, and their ratio and the risk of a first stroke: A meta-analysis and case-control study. Metab. Brain. Dis. 30, 1319-1330 (2015).

30. Sierra-Johnson, J. et al. ApoB/apoA-I ratio: An independent predictor of insulin resistance in US non-diabetic subjects. Eur. Heart. J. 28, 2637-2643 (2007).

31. Fritzen, A. M. et al. ApoA-1 improves glucose tolerance by increasing glucose uptake into heart and skeletal muscle independently of AMPKa(2). Mol. Metab. 35, 4 (2020).

32. Watts, G. F., Ooi, E. M. \& Chan, D. C. Therapeutic regulation of apoB100 metabolism in insulin resistance in vivo. Pharmacol. Ther. 123, 281-291 (2009).

33. Skogsberg, J. et al. ApoB100-LDL acts as a metabolic signal from liver to peripheral fat causing inhibition of lipolysis in adipocytes. PLOS ONE 3, 20 (2008).

34. Lee, J. Y. et al. Apolipoprotein B binds to enolase-1 and aggravates inflammation in rheumatoid arthritis. Ann. Rheum. Dis. 77, 1480-1489 (2018).

35. Mente, A. et al. Association of dietary nutrients with blood lipids and blood pressure in 18 countries: A cross-sectional analysis from the PURE study. Lancet. Diabetes. Endocrinol. 5, 774-787 (2017).

\section{Acknowledgements}

We thank all staffs and participants involved in this study.

\section{Author contributions}

J.X. conceived and designed the research; H.D., W.N., Y.B., X.Y., Y.Z., H.Z. and Y.S. collected the data; H.D. and W.N. performed the data analysis and drafted this paper, J.X. critically revised the manuscript. J.X. had primary responsibility for final content. All authors read and approved the final manuscript.

\section{Funding}

This work was partly supported by the 2020 Innovation and Entrepreneurship Program of Jiangsu Province (Doctor Funds of the Innovation and Entrepreneurship Program), the Nantong Health Committee Foundation (No. QA2020031), the Science and Technology Planning Project of Shenzhen City, Guangdong Province, China (No. SZGW2018002), Sanming Project of Medicine in Shenzhen (No. SZSM201811093), the Science and Technology Planning Project of Shenzhen City, Guangdong Province, China (No. JCYJ20180703145202065), the Science and Technology Planning Project of Shenzhen City, Guangdong Province, China (No. KCXFZ20201221173600001), and Shenzhen Medical Key Discipline Construction Fund. The funders had no role in the design, analysis or writing of this article.

\section{Competing interests}

The authors declare no competing interests. 


\section{Additional information}

Supplementary Information The online version contains supplementary material available at https://doi.org/ 10.1038/s41598-022-06829-w.

Correspondence and requests for materials should be addressed to J.X.

Reprints and permissions information is available at www.nature.com/reprints.

Publisher's note Springer Nature remains neutral with regard to jurisdictional claims in published maps and institutional affiliations.

(c) (i) Open Access This article is licensed under a Creative Commons Attribution 4.0 International License, which permits use, sharing, adaptation, distribution and reproduction in any medium or format, as long as you give appropriate credit to the original author(s) and the source, provide a link to the Creative Commons licence, and indicate if changes were made. The images or other third party material in this article are included in the article's Creative Commons licence, unless indicated otherwise in a credit line to the material. If material is not included in the article's Creative Commons licence and your intended use is not permitted by statutory regulation or exceeds the permitted use, you will need to obtain permission directly from the copyright holder. To view a copy of this licence, visit http://creativecommons.org/licenses/by/4.0/.

(C) The Author(s) 2022 